\title{
Cooperative Firing Activity in Simultaneously Recorded Populations of Neurons: Detection and Measurement ${ }^{1}$
}

\author{
GEORGE L. GERSTEIN, ${ }^{\star, 2}$ DONALD H. PERKEL, $\ddagger$ AND JUDITH E. DAYHOFF§
}

*Departments of Physiology and of Biophysics, School of Medicine, University of Pennsylvania, Philadelphia, Pennsylvania 19104, $\ddagger$ Department of Psychobiology, University of California, Irvine, California 92717, and $§$ Judith Dayhoff and Associates, P. O. Box 4029, Mountain View, California 94040

\begin{abstract}
Recent advances in techniques for chronic recording from multiple extracellular microelectrodes allow simultaneous observation of firings of substantial populations of neurons. We describe a new conceptual representation of cooperative behavior within the observed neuronal population. This representation leads to a new technique for detecting and studying functional neuronal assemblies that are characterized by temporally related firing patterns. The representation may be applied to both dynamic and long-term aspects of cooperativity. The basic idea is to map activity of neurons into motions of particles in a multidimensional Euclidean space. Each neuron is represented by a point particle located in this space. In the simplest version of the mapping, each nerve impulse results in an increment in a "charge" associated with that particle; between firings the charges decay. The force exerted by any such particle on any other is, by analogy with some physical forces, proportional to the product of their charges and may depend on the Euclidean distance separating them. The force on a particle directly affects its velocity rather than its acceleration, as with actual particles in a viscous medium. These forces result in aggregation of those particles that correspond to neurons tending to fire together; separate clusters represent independent cooperative groups. Modification of the charges and forces permits inclusion of inhibitory interactions. Identification, measurement, and display of the resulting clusters can be performed with any of a number of algorithms. We illustrate the application of this approach to populations of computer-simulated neurons having both direct and indirect excitatory coupling.
\end{abstract}

A popular experimental paradigm in the study of brain function, used extensively for the last two decades, is based on electrophysiological recording of single neuron activity. Such work examines neural firing patterns as a function of stimulus, motor, or behavioral conditions and generally is used to measure a receptive (or motor) field or a tuning curve for the neuron. Many such single neuron measurements, carried out sequentially, together with various modes of anatomical localization, are then used to infer the properties of

Received March 16, 1984; Revised September 24, 1984;

Accepted October 3, 1984

${ }^{1}$ This work was supported by National Institutes of Health Grants NS 05606 (G. L. G.), NS 16451 (D. H. P.), and NS 17754 (D. H. P.), and by System Development Foundation Grant SD00013 (G. L. G.). We thank Dr. A. Aertsen for helpful discussions

${ }^{2}$ To whom correspondence should be addressed an assembly of neurons in the system under study. Although such measurements allow examination of firing patterns down to a time scale of milliseconds, they provide no direct evidence about the functional connectivity among the sequentially observed neurons. This must be done indirectly by inference based on shared or hierarchical response properties of the several neurons. Similarly, the role of the neuronal assembly in the information handling (stimulus analysis or behavioral) process can only be viewed in terms of the properties of the individual neurons. A striking result of such work by Mountcastle (1957), Hubel and Wiesel (1962), and others has been the discovery of widespread modularity in many portions of the nervous system; the modularity is based on the spatial clustering of neurons with similar stimulus response properties (for example, cortical columns or slabs). However, the internal operations and dynamic aspects of connectivity within these modular assemblies remain largely unknown because, so far, only sequential measurement has been employed.

Direct investigation of neuronal assemblies, of their possibly time varying organization and extent, and of their dependence on stimulus or behavioral circumstances, can only be based on simultaneous but separable observation of many individual neurons. Such work involves a number of additional technical and conceptual difficulties (reviewed in Gerstein et al., 1983), but is becoming increasingly practical. Thus, it is now becoming possible to implant chronic multiple microelectrodes or to use analogous optical techniques (Grinvald et al., 1981) to record the spatiotemporal patterns of firing in a substantial population of neurons. These powerful experirnertal techniques, together with the appropriate analyses, should allow detailed study of cooperativity among subpopulations of neurons as well as the relation of such cooperativity to experimental and behavioral parameters.

However, the very profusion of data produced in such experiments creates serious problems of analysis and interpretation; these problems are conceptual as well as practical. The principal conceptual problems are (1) defining cooperativity or functional grouping among neurons and (2) formulating quantitative criteria for recognizing and characterizing such cooperativity. The chief practical problem is screening the massive data base in order to detect and identify putative neuronal groups as defined through measures like temporally related firing. The goal is to measure the extent and nature of the cooperativity within each group, the degree of independence from other such groups, the relationship to known modularity and individual neuron response characteristics, and the relationship of group properties to experimental circumstances.

One hopes ultimately to relate such measures to the underlying physiological mechanisms that select and modulate dynamic group properties. Although such mechanisms are, undoubtedly, ultimately based on synaptic modulation and its biophysical substrates, one may speculate that mechanisms affecting groups of neurons will themselves involve activity of groups of neurons. Thus, we probably 
need to understand groups of neurons interacting with other groups of neurons.

It should be stressed that the principal tools available to date for the examination of rclated firing among neurons involve crosscorrelation of the firing of two or at most three neurons at a time (reviewed in Gerstein et al., 1983). These tools allow inference of effective connectivity and have been used by many investigators in many parts of the nervous system. If, however, we record from populations of 10 or more neurons, such pairwise analysis becomes exceedingly tedious simply because of combinatorial arithmetic. In addition, neuronal group properties must then be inferred from combinations of neuronal pair properties, a process that is subjectively difficult. We need theoretical means to treat a neuronal group as a single entity.

We have previously addressed certain problems of neural groups (Gerstein et al., 1978). Conceptually, the new approach (which is related to the gravitational clustering technique of Wright, 1977) is clearer than the older method and entails minimal reliance on subjective analysis. It provides greater flexibility in the criteria for related firing of two or more neurons. We emphasize that the new approach deals with a neuronal assembly as an entity rather than as a set of neural pairs; it differs fundamentally from analysis by pair cross-correlation methods (Perkel et al., 1967). Quasi-on-line implementation is possible, so that experimental strategy can be modified while the neural assembly is still under observation.

After describing the basic approach together with some modifications that enhance performance of the calculations, we examine some simple neuronal circuits whose firing behavior was simulated on a computer. Finally, we indicate some generalizations of the method that make accessible other aspects of neuronal cooperativity.

\section{The Basic Method}

Representation of neurons as point particles. We begin by representing each of $N$ neurons by a fictitious particle, located at a point in $\mathrm{N}$-dimensional Euclidean space. At any time, then, the vector position of the particle corresponding to neuron $i$ is written as

$$
x_{1}=\left(x_{i 1}, x_{i 2}, x_{i 3}, \ldots, x_{i N}\right)
$$

where $x_{i j}$ is the $j$ th coordinate value for particle $i$.

Initially, all of the particles are set to be equidistant. Specifically, each particle is located at a vertex of an $N$-dimensional hypercube or, most simply,

$$
\begin{aligned}
x_{1} & =(\sqrt{2 / 2}, 0,0, \ldots) \\
x_{2} & =(0, \sqrt{2 / 2}, 0, \ldots) \\
x_{3} & =(0,0, \sqrt{2 / 2}, \ldots) \\
& \ldots \\
x_{N} & =(0,0,0, \ldots, 0, \sqrt{2 / 2})
\end{aligned}
$$

The initial distance between any two particles is then equal to 1 .

For the calculations shown in this paper, we have scaled the initial distance between any two particles to be 100 .

Dynamics of charges. When a neuron fires, its particle acquires a finite increment of a fictitious "charge," analogous to an electrical or gravitational charge. Between firing times the charge on particle $i$ obeys the differential equation

$$
d q_{i} / d t=-q_{i} / \tau
$$

for which the solution is

$$
q_{,}(t)=q_{1}(0) e^{-t / \tau}
$$

in which $t$ is measured from just after the last augmentation. The time constant $\tau$ is a parameter chosen by the investigator. The transformation from firing history to charge is illustrated in Figure 1.

In the simplest version of this scheme, the particles attract each other with a force proportional to the product of their charges. Note

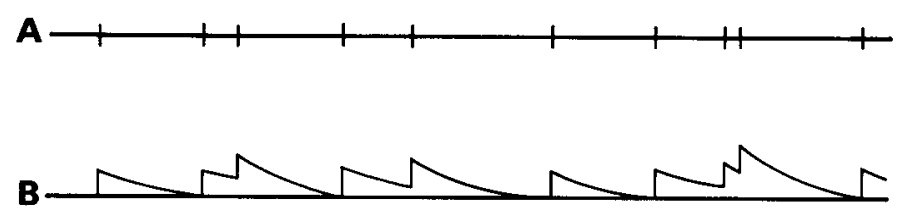

Figure 1. Conversion of spike train $(A)$ to charge $(B)$ used in the gravitational calculation. At the time of each neural impulse, the existing charge in the representation of that neuron is incremented by a fixed amount. The magnitude of this charge increment is different for each neuron and is inversely related to its average neuronal firing rate. Between neural impulses the charge decays with a fixed time constant (same for all nourons)

that in contrast with electricity and gravitation, the charges fluctuate; they decay continually and are augmented at the instants the corresponding neurons fire. The force may depend on distance between particles. The individual forces exerted on a particle add vectorially, and the particles move under the influence of the chang ing forces according to a particular dynamic law; the most convenient one here is comparable to motion through a viscous medium.

As a result of the fluctuating charges and attracting forces, the particles representing any group of neurons that tend to fire simultaneously will exert mutually attractive forces and will be drawn closer together. After a sufficient time these sets of synchronously firing neurons will be represented by clusters of particles in $\mathrm{N}$-space. The clusters can then be identified, distinguished, and characterized by standard algorithms of cluster analysis.

Propulsive fields. The propulsive field produced by particle $j$ at the location currently occupied by particle $i$ has the normalized unit direction in $N$-space given by

$$
r_{1}(j)=\left(x_{i}-x_{i}\right) / s_{i j}
$$

where $s_{i j}$ is the Euclidean distance between particles $i$ and $j$ :

$$
s_{i j}^{2}=\left\|x_{1}-x_{1}\right\|^{2}=\sum_{k=1}^{N}\left(x_{j k}-x_{i k}\right)^{2}
$$

The amplitude of the propulsive field is proportional to the charge on $j$ and may fall off with distance by a dimensionless attenuation function $A\left(s_{i i}\right)$. (The most familiar such function would be an inversesquare law, $\left.\left(s_{o} / s_{i j}\right)^{2}\right)$.

In general, then, the propulsive vector field at point $i$ exerted by all other particles is given by

$$
\boldsymbol{f}_{\boldsymbol{i}}=\sum_{j \neq i} q_{j} \boldsymbol{r}_{\boldsymbol{i}}(j) A\left(\boldsymbol{s}_{i j}\right)
$$

We have found that elimination of the attenuation factor is convenient and desirable, and thus we have set $A\left(s_{i i}\right)=1$.

Motion under the propulsive field. The force vector acting on a particle is proportional to the product of its charge and the propulsive field, i.e., to $q \boldsymbol{f}_{i}$. Although it would be possible to imagine the particles as being accelerated by these forces, like point masses in a gravitational field, this would involve second-order differential equations. Since we are not concerned with a realistic prediction of trajectories of fictitious particles in a hypothetical $\mathrm{N}$-dimensional space, but rather with ensuring that attractive forces will induce particles to approach one another, the simplest dynamic rule is to imagine the particles as moving in a viscous fluid. We also assume that they always travel at terminal velocity, which is proportional to the force exerted. With these assumptions, the dynamic equation for particle $i$ is simply

$$
d x_{1} / d t=\sigma q_{i}(t) f_{i}(t)
$$

in which the single scalar parameter $\sigma$ subsumes the amplitude of the force, the reciprocal of the "fluid" viscosity, etc. The dimension of $\sigma$ is velocity per squared unit of charge.

It may be noted that the points do not move in an arbitrary fashion throughout the $N$-space. In fact, they are initially placed on a hyperplane of dimension $N-1$, and will henceforth remain on that 

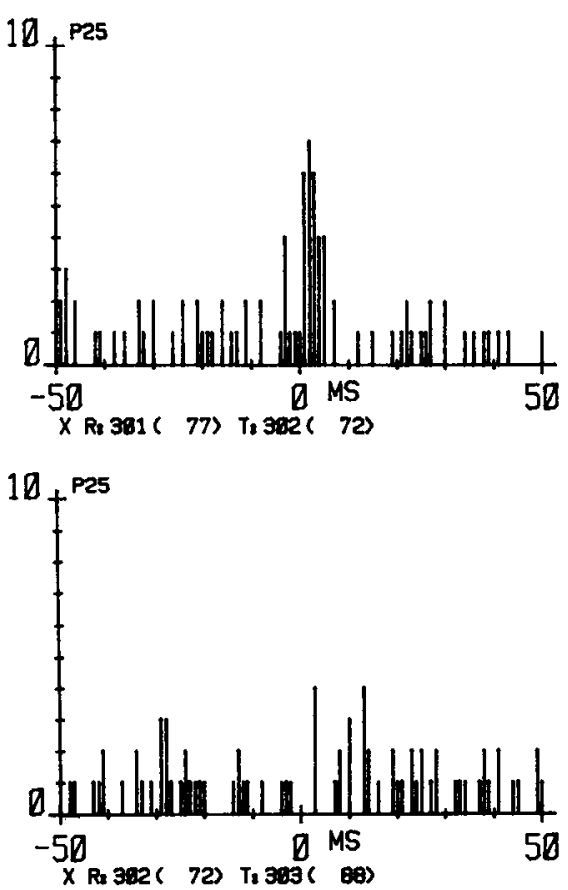
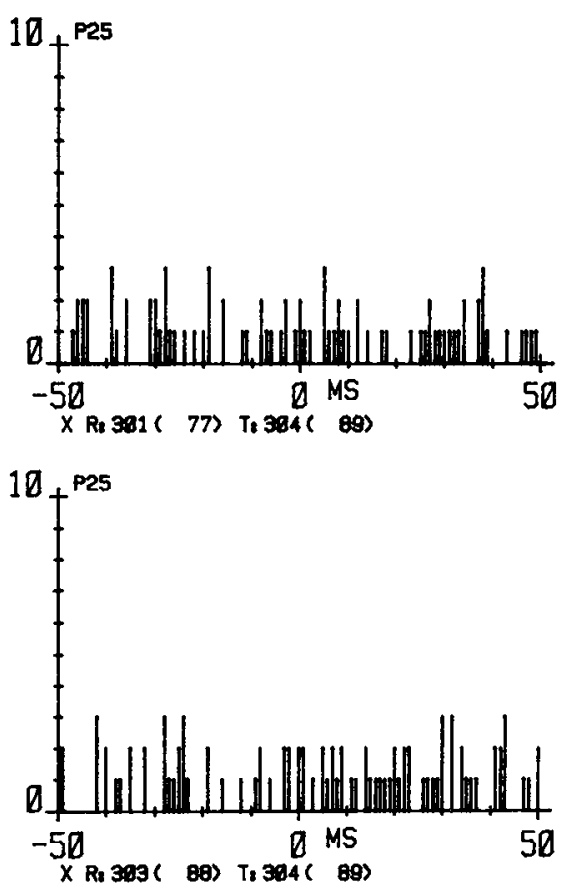

Figure 2. Cross-correlograms for a group of spike trains that were produced by computer simulation. These and similar spike trains are analyzed in Figures 3 and 4 . Each group of data contains 10 spike trains, each Poisson like, with a mean rate of $10( \pm 2)$ spikes/ sec. Neuron 1 has an excitatory synaptic connection to neuron 2 (replication probability 0.25 ); the other eight neurons are completely independent. Total data length is $8.5 \mathrm{sec}$, with 70 to 100 spikes in each train. The cross-correlogram in the upper left is for the synaptically coupled neuron pair; the other three correlograms are typical for independent neuron pairs. (There are 41 other similar histograms between the other independent pairs).

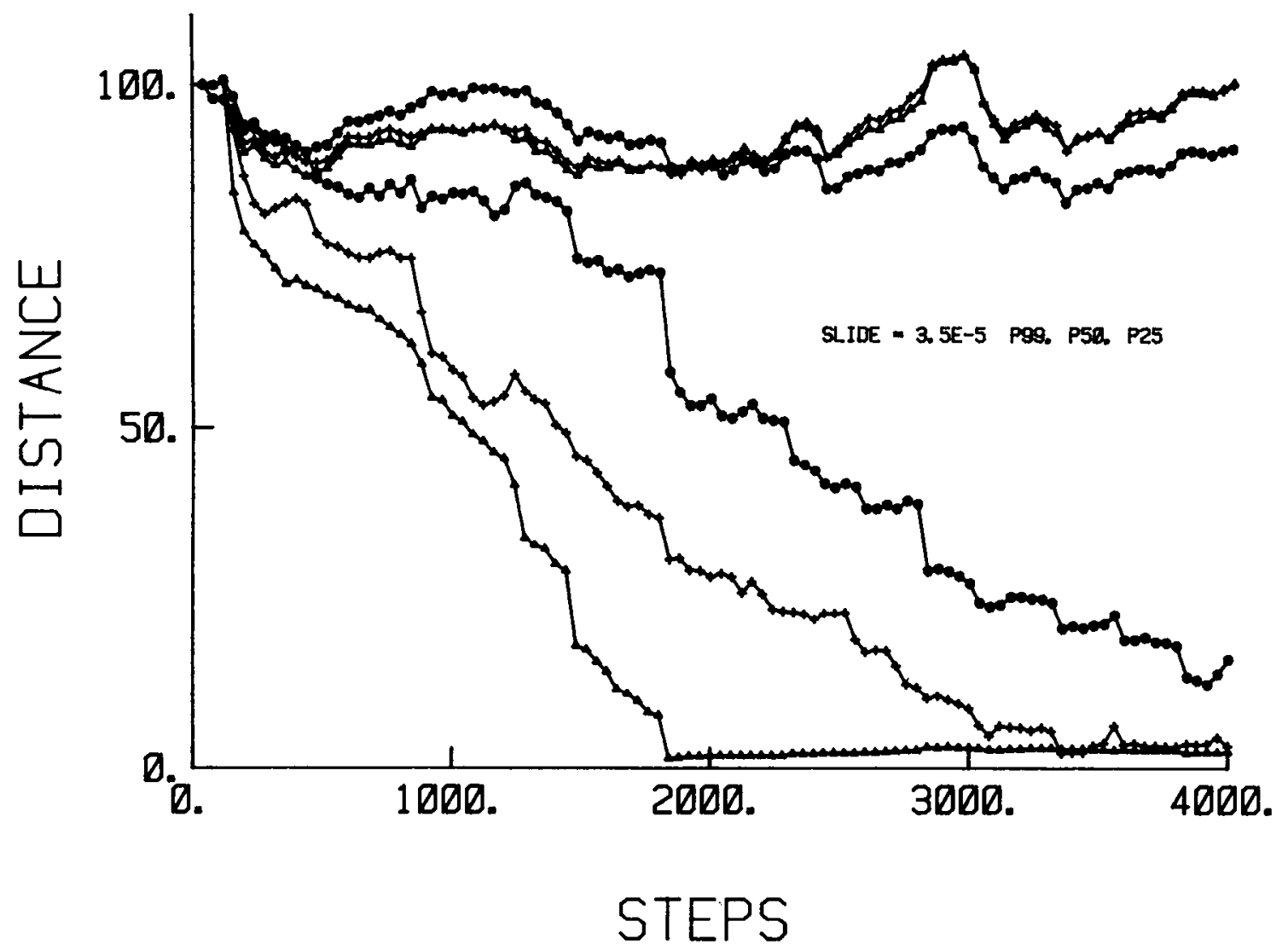

Figure 3. The time to gravitational aggregation decreases as the strength of synaptic connection increases. Distance between selected pairs of points is shown as a function of step number (time) in the gravitational calculation. All pairs start at the distance of 100 arbitrary units; each step in the calculation corresponds to $2 \mathrm{msec}$ of laboratory time. Time constant for charge decay was set at $10 \mathrm{msec}$, and the distance moved per unit force was $3.5 \times 10^{-5}$ units. The three descending curves show distances between points that represent neurons connected with (left to right) decreasing synaptic strength (replication probabilities $0.99,0.50,0.25$, respectively). Distances between typical points representing independent neurons are at the top of the figure and remain essentially unchanged as the computation progresses.

hyperplane. In the discussions and calculations of this paper we have not made use of this slight reduction of dimensionality, and have described the movements in the full $N$ space. We believe this convention simplifies the initial visualization of our problem as well as its initial mathematical formulation. We may anticipate some small improvement in computation time if the calculation is expressed in the $N-1$ dimensional subspace.

Having set up the explicit set of ordinary differential equations (8), 


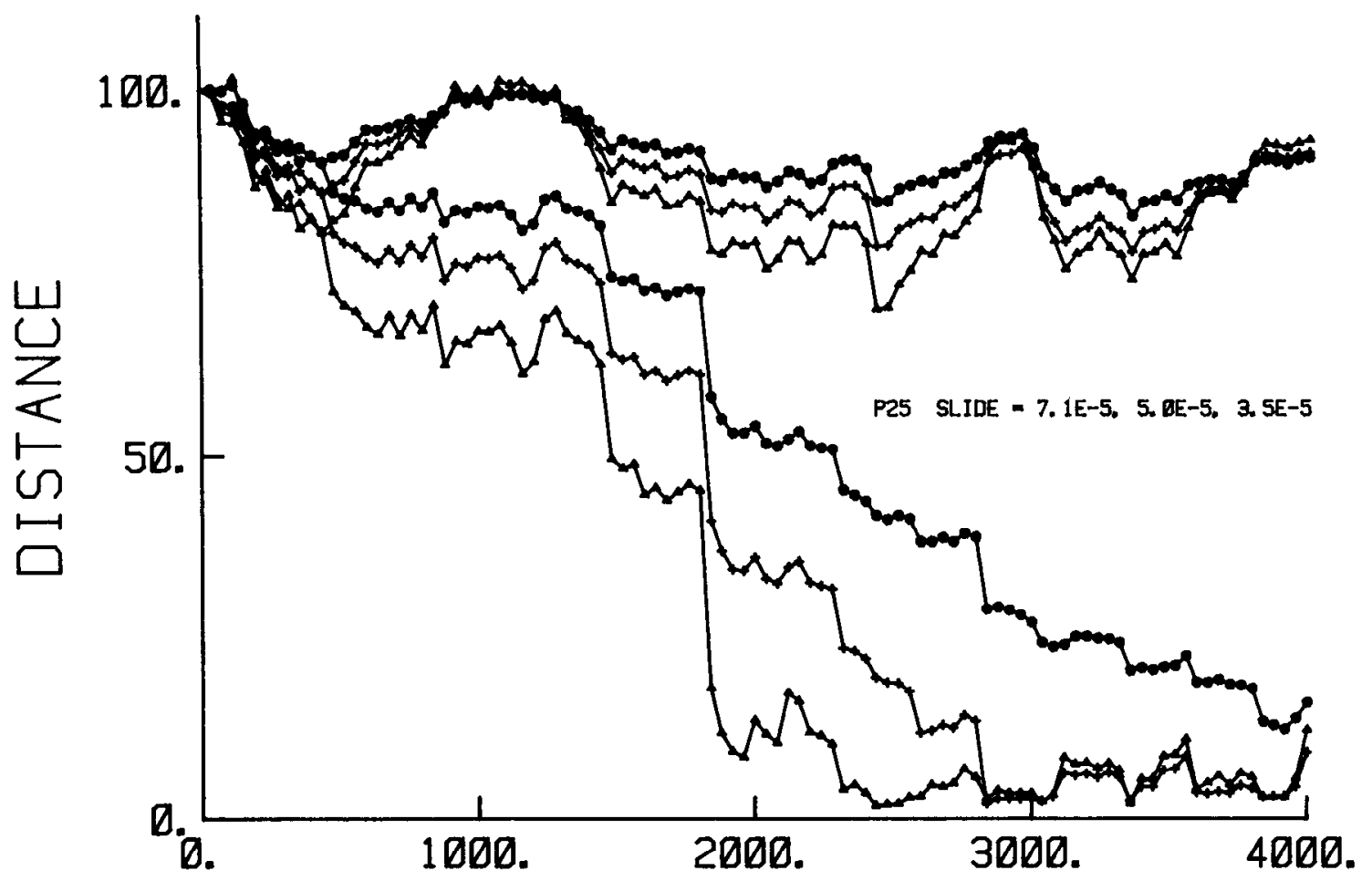

\section{STEPS}

Figure 4. The time to gravitational aggregation decreases as the distance moved per unit force increases. Axes are as in Figure 3. The three descending curves represent recomputations of the data with weakest synaptic connection (replication probability 0.25 ) shown in Figure 3 , with three different values for the constant $\sigma$. From lett to right, $\sigma=7.1 \times 10^{-5}, 5.0 \times 10^{-5}$, and $3.5 \times 10^{-5}$, respectively. Distances between points representing a typical pair of independent neurons are shown at the top for the same three calculation parameters. Note the increase in variance as the value of $\sigma$ increases.

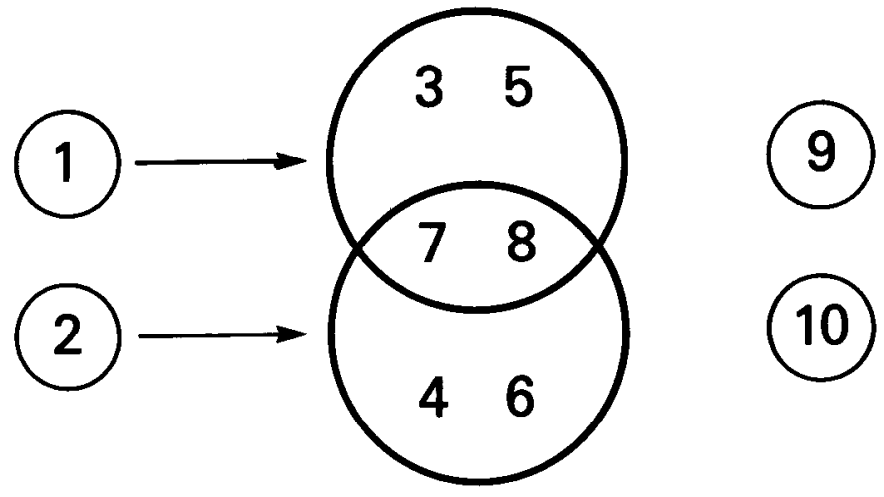

Figure 5. A Venn diagram of more complicated neuronal connectivity that was simulated in a set of 10 spike trains. These data are analyzed in Figures $6,7,8$ and 9 . Neurons 1 and 2 are drivers. Neurons 7 and 8 receive input from both drivers, whereas neurons 3,5 and 4,6 receive input only from one driver. Neurons 9 and 10 are independent.

we can use any standard numerical integration scheme to follow the positions of the particles in response to the recorded history of firing patterns of the corresponding neurons. We emphasize again that we are not concerned with dynamic realism but are using these dynamics only as a means to allow the appropriate particles to aggregate; thus, the simplest algorithm suffices. Using Euler integration with a time step $h$, we have the simple formula

$$
\mathbf{x}_{\mathbf{1}}(t+h)=\mathbf{x}_{\mathbf{1}}(t)+h \sigma q_{i}(t) \boldsymbol{f}_{\boldsymbol{\prime}}(t)
$$

We note that in this simple version there are two essential parameters that may be manipulated by the investigator. The time constant for decay of charge, $\tau$, governs the strictness of the degree of synchrony required to produce aggregation. The other parameter, $\sigma$, may be thought of as a measure of mobility of particles and can be varied to modulate the rate of coalescence.

\section{Modifications to the Algorithm}

Independence of rate. In the representation described, in which every particle receives the same increment of charge when its associated neuron fires, the propulsive influence of that particle is proportional to the neuron's mean firing rate. Such a rate dependence may not be desirable and can be eliminated by a simple convention. We set the increment of charge added at the time of each action potential to be the mean interval (the reciprocal of $\rho$, the mean firing rate) for the neuron represented by that particle. It is then easy to show that the mean value of charge is the same for all particles, and, for exponential decay of charge, is in fact numerically equal to $\tau$, the time constant for decay of charge. Other types of rate normalization are possible and will be contrasted and evaluated elsewhere.

Repulsion and attraction. Another undesirable property of the original formulation is that all particles attract and will eventually aggregate. In other contexts, this phenomenon has been used directly; Wright (1977) has used time until aggregation to define clusters. For our application, it is preferable that the particles corresponding to independently firing neurons neither attract nor repel one another on the average. This can be accomplished by replacing the charge $q$ in equations 7 to 9 by an effective charge $(q-\theta)$, where $\theta$ is a reference level of charge, so that the force between particles $i$ and $j$ will be proportional to $\left(q_{i}-\theta_{i}\right)\left(q_{j}-\theta_{j}\right)$. If both neurons have recently fired, so that both charges are above their reference levels, the particles will attract. If one neuron has not fired 

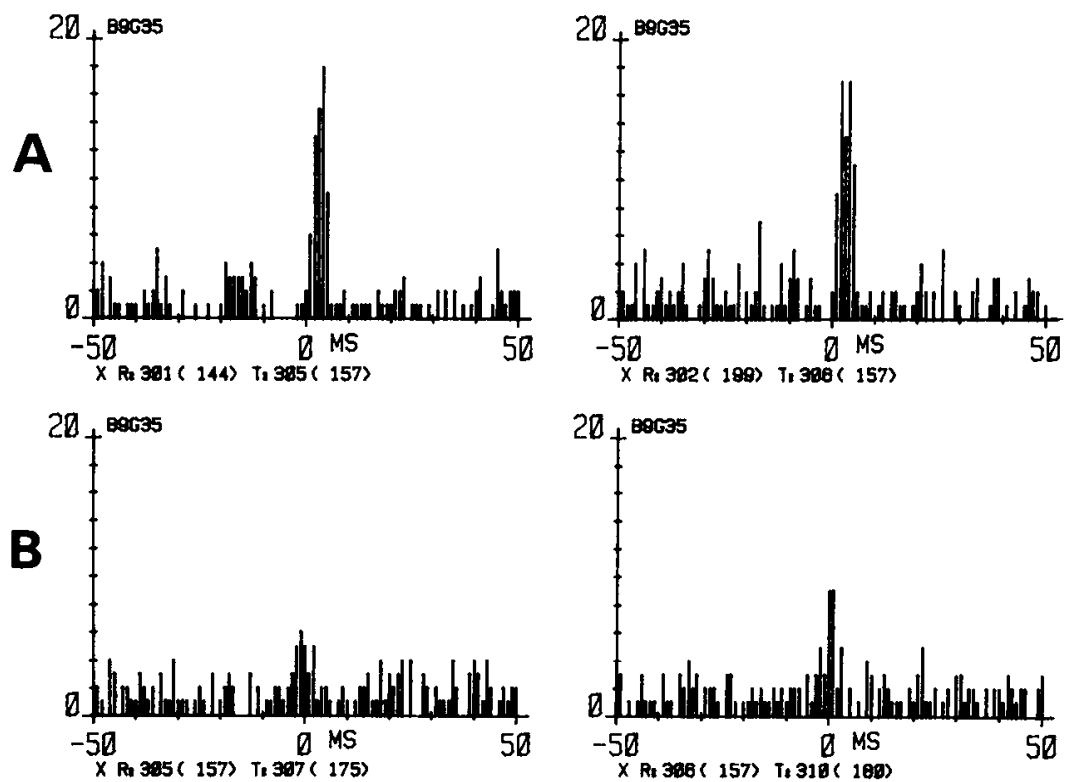

Figure 6. Typical cross-correlograms of spike trains from computer simulation of the neural assembly shown in Figure 5. All 10 spike trains were Poisson like (mean rates, $10 \pm 2$ spikes/sec; duration, $17 \mathrm{sec}$ ). Synaptic connections were simulated by replication probability of 0.35 . $A$, Two neuron pairs, each consisting of driver and driven. $B$, Two neuron pairs each consisting of two neurons driven from the same driver. $C$, Two pairs of independent neurons.
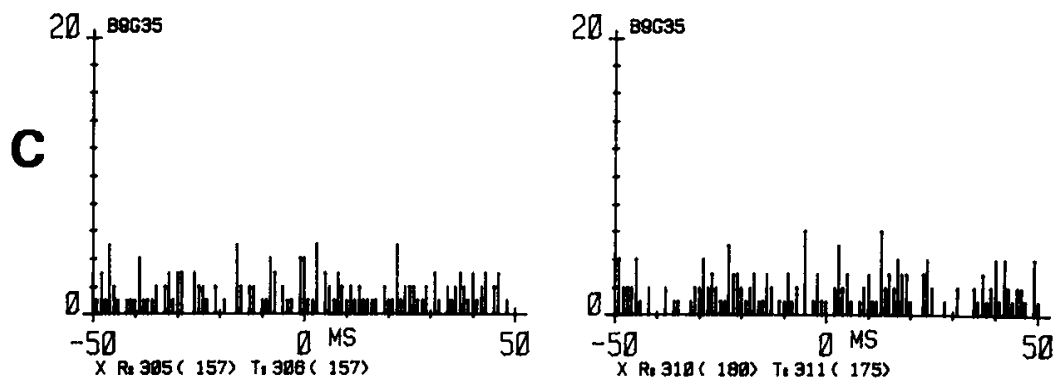

recently and the other has, the corresponding particles will repel. Finally, if neither neurons has fired recently, so that both charges are below their reference levels, the particles will attract weakly. These are precisely the properties needed to ensure aggregation of those particles whose neurons tend to fire synchronously and, more weakly, of those particles whose neurons tend to be silent synchronously.

The appropriate value for the reference charge $\theta$ is the mean value of charge for that neuron. If, as suggested above, the increment of charge has been set equal to the mean firing interval of the corresponding neuron, then the mean charge is the same for all particles, and, if charge decays exponentially, is numerically equal to $\tau$. The net effect of these modifications is that $q$ is replaced by $(q-\tau)$ in equations 7 to 9 :

$$
\begin{gathered}
\boldsymbol{f}_{\boldsymbol{i}}=\sum_{j \neq j}\left(q_{i}-\tau\right) \boldsymbol{r}_{i}(j) \\
\mathbf{x}_{\boldsymbol{i}}(t+h)=\mathbf{x}_{\boldsymbol{i}}(t)+h \sigma\left[q_{i}(t)-\tau\right] \boldsymbol{f}_{\boldsymbol{i}}(t)
\end{gathered}
$$

Close approaches. A numerical complication of this model arises when the distance between any two particles becomes small. Euler integration and similar techniques give rise to unwanted oscillations of closely neighboring particles. This is especially severe when using a spatial attenuation function having a singularity at the origin, such as the inverse square law. A simple remedy is to introduce a minimal distance between particles within which the net force is zero. Note that, once two particles are within this minimal distance and cease to interact, they continue to be influenced by more distant particles through the firing of their corresonding neurons. A continuous spatial attenuation function, which gradually goes to zero at the origin, may also be used.

\section{Results}

Factors affecting the aggregation process. The speed and variability in the aggregation of particles corresponding to interacting

neurons depend on the strength and nature of the interaction and on the choice of parameters used in the computations. We illustrate these effects with computer simulation of a simple neuronal circuit consisting of a pair of synaptically connected neurons together with a set of independently firing control neurons.

In the simulations all independent impulse trains were Poisson processes. The excitatory synaptic interaction was approximated by choosing impulses at random in the presynaptic train, with a specified probability, and copying each selected impulse to the postsynaptic train, imposing a delay chosen from a suitable probability distribution. In addition, the nearest following original impulse to the copied impulse in the postsynaptic train was deleted in order to preserve mean firing rate. Thus, the postsynaptic impulse train contained a partial, noisy, delayed replica of the presynaptic train. This class of spike train simulator is, or course, only intended to accurately model spike trains with temporal interrelationships similar to those found in actual recordings; it is not intended to model the underlying integrative dendritic processes.

Mean firing rates were between 8 and 12 impulses/sec; the sample was $8.5 \mathrm{sec}$ long. The delay function was a rectangular probability distribution from 1 to $5 \mathrm{msec}$. Cross-correlation histograms are shown in Figure 2 for a replication probability of 0.25 .

In Figure 3, we illustrate the effect of strength of synaptic interaction on the aggregation process. The strength was varied by modulating the replication probability, using values of $0.25,0.50$, and 0.99 . The strongest interaction corresponds to a complete but temporally noisy replication of the presynaptic impulse train. The figure shows, for each case, the time coursc of the distance between the points corresponding to the pre- and post-synaptic neurons as well as those of an unrelated control pair. It is clear that, for unrelated neurons, the points execute a random walk while remaining at an approximately constant distance. The points for an interacting pair, on the other hand, approach each other consistently although somewhat irregularly. The average approach velocities increase with 


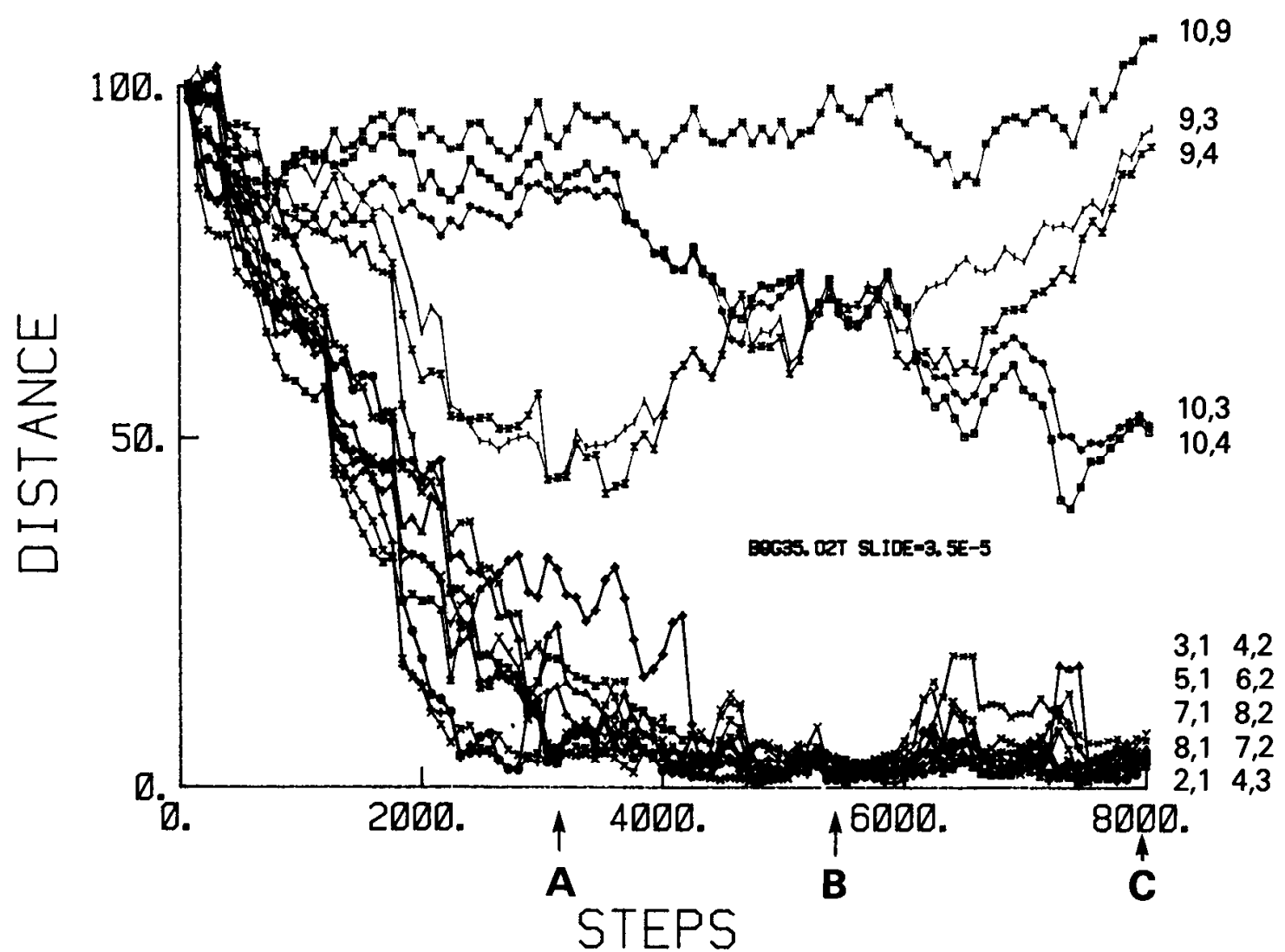

Figure 7. Gravitational computation for the 10-spike train data set described in Figures 5 and 6 . Ordinate, Distance between points representing selected neuron pairs; abscissa, steps in the computation (2 msec/step). Three step (or time) values labeled $A, B$, and $C$ are selected on the abscissa for reference to Figure 8. See the text.

increasing strength of synaptic interaction; therefore, the two points coalesce most rapidly for the strongest interaction. In the case of strongest interaction, coalescence was achieved after about 40 impulses (4 sec).

In addition to the intrinsic strength of the neuronal interaction, the rate of coalescence is influenced by the parameters of the computation. In particular, the velocity parameter strongly affects the rate of coalescence for a given pair of interacting neurons as well as the degree of random fluctuation in distance between each pair of points. These effects are shown in Figure 4, in which the impulse trains incorporating the weakest interactin $(0.25$ probability of replication) were analyzed using three different values of the velocity parameter.

Another important factor influencing the aggregation process is the time constant for decay of charge. If the time constant is chosen to be small, aggregation will result only for interactions having correspondingly short latencies and time jitter. As the time constant increases, interactions having longer latencies and/or greater jitter will be included in the aggregation process. If, however, the time constant becomes comparable to average intervals between impulses, sensitivity and selectivity to temporal interactions are effectively lost. If nothing is known about the nature of interactions among the neurons under study, therefore, it is highly advisable to analyze the data several times, using different values of the time constant as appropriate.

The two other parameters that must be chosen for the computation are the time step $h$ and the approach distance below which the attractive force is set to zero. Clearly, the time step must be smaller than the time constant for decay of charge. The critical approach distance should be greater than the maximum increment in distance in a single time step of the computation, which is given approximately by

$$
\Delta x_{\max } \simeq h \sigma / \rho_{\min }^{2}
$$

where $\rho_{\text {min }}$ represents the minimum firing rate over the ensemble of neurons. Choice of this critical distance may be used as a criterion for significant interaction; judicious use of such a criterion at a value larger than that given by equation 12 can save computation time.

Neuronal population containing several types of interaction. A more complex and extensive example is provided by applying the algorithm to a set of neurons more diversely connected than in the previous example. Here we use a computer simulation of 10 neurons, consisting of (a) two independently firing presynaptic neurons, (b) a set of three postsynaptic neurons for each presynaptic one, one neuron in each of these sets being driven by both presynaptic neurons, and (c) two independently firing control neurons. This example provides direct synaptic interaction as well as two degrees of shared input, as seen in the schematic circuit (Fig. 5). The impulse trains were generated by the same rules as in the previous example; the sample was made $17 \mathrm{sec}$ long having up to 200 impulses for each neuron. Typical cross-correlation histograms are shown in Figure 6.

Once again, we illustrate the aggregation process through plots of pairwise distances as a function of time. The identities (see Fig. 5) of the several pairwise distances are indicated at the right of Figure 7 and fall into four groups. At the top, the distance between points 9 and 10 (which represent the control neurons) remains essentially unchanged throughout the calculation. Next from the top are the distances for 9,3 and 9,4 (representing in each pair one control neuron and one postsynaptic neuron); these distances initially decrease, but later increase. The third group from the top are the distances for 10,3 and 10,4 (the other control neuron and one postsynaptic neuron); these distances slowly decrease through the calculation. Finally, at the bottom are the pairwise distances for various transynaptic pairs, as well as for the two drivers $(1,2)$ and 


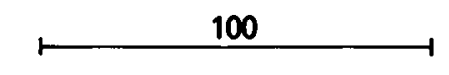

(9)

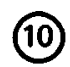

Time A

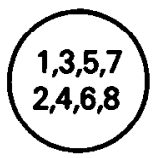

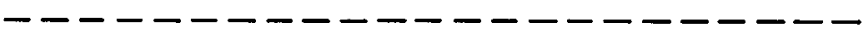

(9)

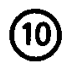

Time B

$1,3,5,7$

$2,4,6,8$

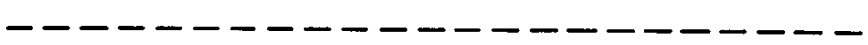

(9)

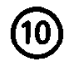

Time C

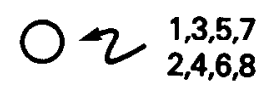

Figure 8. Approximate projection of the 10 points (representing the 10 neurons) from the 10-space onto an appropriate plane. The projection is shown at the three times $(A, B$, and $C$ ) identified in Figure 7 . The scale showing a distance of 100 is at the top. At Time $A$ the group of points representing interacting neurons has not yet fully aggregated; however, the loose group is closer to point 9 than to point 10 (these represent the independent neurons). At Time $B$ the aggregation is complete, and equidistant from points 9 and 10. At Time $C$ the tight aggregation has drifted closer to point 10.

for an independent postsynaptic pair $(4,3)$; all of these distances demonstrate similar rapid coalescence.

The reason there is no distinction in the behavior of any of these pairs is that all synaptic interactions have been made the same strength. Thus, each driver attracts equally (and is attracted by) each of its postsynaptic partners. This strong interaction will indirectly bring all of the postsynaptic partners of the same driver close to each other, even though their own direct interaction is weaker. Since neurons 7 and 8 receive input from both drivers, they will serve as an attractant for the groups of points aggregating around each driver, thus accounting for the eventual proximity of 1,2 (independent drivers) and 3,4 (independent postsynaptic pair). Thus, pairwise distances can identify a subset of interacting neurons but are not in themselves sufficient to infer the circuit or its relative synaptic strengths.

The pairwise distances shown in Figure 7 can be interpreted in terms of clustering of the points moving in the representational space of the calculation. This is most readily done by projecting the positions of our 10 points as they move in the 10 space onto some appropriate plane. (Considerations and methods of choosing such a projection plane will be discussed elsewhere). Note at the bottom of Figure 7 that we have marked three times with letters $A, B$, and C. At time $A$ (Fig. 8, top) we see that the projected points representing interacting neurons have loosely coalesced, and that this rather large cluster is nearer to point 9 than to point 10 (these two points represent the independent control neurons). At time $B$ (Fig.
8 , middle), the points representing the interacting neurons have coalesced into a tight cluster which is about halfway between the two points which represent the control neurons. Finally, at time $C$, the tight cluster has drifted closer to point 10 than to point 9 .

The results shown in Figures 7 and 8 were obtained using the impulse trains from the two presynaptic neurons. Clearly, a typical physiological recording may fail to include signals from the neurons presynaptic to the recorded neurons. Figure 9 shows the results of analyzing the same data as in Figures 7 and 8 , but excluding the two presynaptic impulse trains. Although the rate of coalescence is somewhat slower because neuron pairs are now only related by shared input rather than by direct synaptic contact, the interacting neurons can still be clearly distinguished from the control cases. It is striking that omission of the driving neurons from the analysis has so little effect on the selectivity and sensitivity; the same set of interacting neurons is detected in the two examples.

Implementation. The gravity representation was programmed in Fortran and initially run on a Digital LSI 11/23 (RT-11) computer. Under these conditions run times are very unsatisfactory: with typical parameters, analysis of $16 \mathrm{sec}$ of 10-spike trains (as shown in this paper) took almost $3 \mathrm{hr}$. (Note that the calculation time behaves as the third power of the number of neurons). Presumably some small improvements in program efficiency are possible. However, when the same program and data were implemented on a Digital VAX 780 (VMS), run times were $5 \mathrm{~min}$, while on a Data General MV 10000 (AOS/VS), run times fell to 3 min. This is not yet real time but would allow this form of analysis with a short enough time lag to be useful during an experiment. Additional computational speed can probably be obtained with array processor hardware, since the gravity calculation can be reduced to matrix manipulations. (This possibility is worth investigating for laboratory microcomputers as well as the cited minicomputers). At the present stage of development, access to a powerful and unloaded minicomputer seems essential for this problem; the microcomputers inhabiting many neurophysiological laboratories are too slow for practical results.

\section{Discussion}

We have described a method for detecting tendencies toward synchronous firing in groups of neurons. As shown by the examples, the approximate firing synchrony can be produced by a variety of mechanisms, such as direct synaptic input or by shared input. The latter is typically much harder to detect (Perkel et al., 1967).

Although in the examples illustrated we have made use of distances between pairs of points to demonstrate the aggregation process, we must consider the underlying migration of points in the full $\mathrm{N}$-space of this representation. Total information about the behavior of the neuronal population is contained in the time history of the position coordinates of the $N$ points. For $N>3$, this process cannot be directly visualized, and we must resort to a suitable projection or clustering technique.

An intrinsic strength of this representation is that weak neuronal interactions are not masked by strong interactions. The strong direct synaptic interactions reveal themselves at an early stage of the calculations, whereas the more diffuse effects of shared input (assuming the shared driver is off stage) become evident only' after a longer period of observation. The ultimate aggregation of particles in this representation is hierarchically organized, so that strong neuronal interactions rapidly produce tight clusters. These clusters themselves become aggregated more slowly in response to the weaker neuronal interactions. The original tight clusters remain and do not interfere with the aggregation process resulting from the weaker neuronal interactions. Thus, the dynamic evolution of the aggregation can be used to illuminate attributes of the neuronal system. The advantages of observing the dynamic evolution of the aggregations are analogous to the procedures recommended by Wright (1977).

The shapes of the clusters formed in $\mathrm{N}$-space can be important clues to the nature of the underlying neuronal interactions. For 

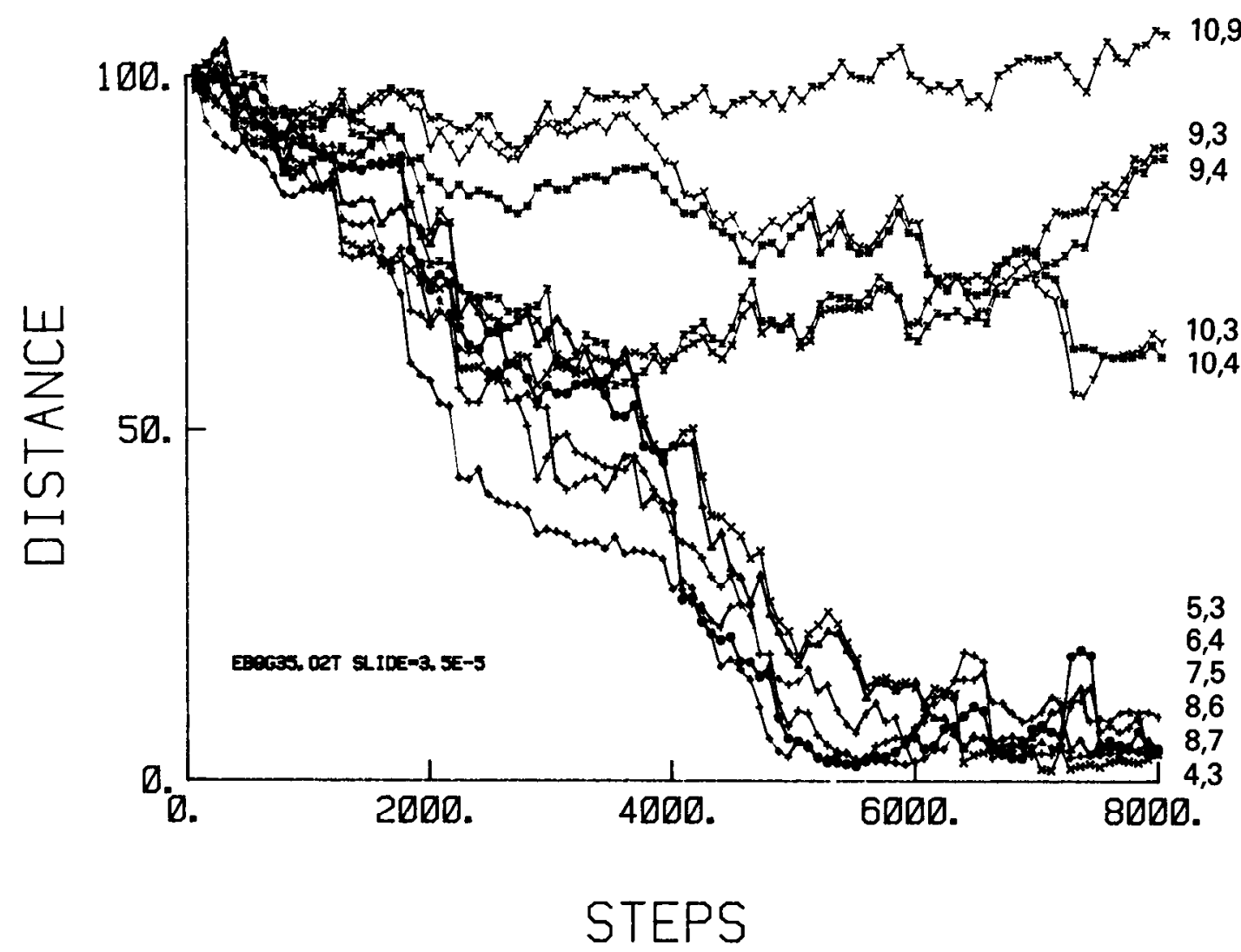

Figure 9. Distances in the gravitational computation for the same data as in Figure 7, but without spike trains 1 and 2 (the drivers). Axes and labels are as in Figure 7. Note that aggregation is slower than in Figure 7, although other features remain the same.

example, if a set of neurons is driven by a single, shared presynaptic excitatory neuron, the corresponding points will form a single cluster. A different set of neurons, similarly excited by a different driver, will form a similar but independent cluster. If there are neurons that receive excitatory input from both drivers, the two clusters will be joined by a bridge, in the fashion of a dumbbell. Points in the neck of the dumbbell correspond to the neurons that receive input from both drivers. This configuration of points resembles a Venn diagram in the $\mathrm{N}$-space.

A different example is provided by a systematic pattern of sequential firing by a set of neurons within the population. Such a sequence might be the result of a chain of synaptically coupled neurons or, alternatively, of an appropriate pattern of delays from a common source of excitation. In the $\mathrm{N}$-space, the resulting configuration of points will resemble a snake or sausage, since there will be aggregation only of pairs of points corresponding to adjacent neurons in the chain. It will be an elongated figure but is unlikely to be a straight line; therefore, detection of such a configuation will require more extensive analysis than needed for more compact or symmetrical clusters.

A useful assessment of significance in the aggregation process can be provided by including one or more impulse trains in the data base that are known to be causally unrelated to any of the other trains. These could be derived from another experiment, or produced artificially on a computer. The corresponding points should remain at approximately a constant distance from all other points, neither aggregating nor separating significantly, as illustrated in our examples.

Up to this point we have assumed implicitly that the pattern of neuronal interactions remains unchanged throughout the period of recording. If in fact the interaction pattern is itself changing, then the consequences of such changes may become apparent in the evolution of the aggregation process. For example, if a strong neuronal interaction ceases to operate, then the corresponding cluster of particles will begin to disperse. If the new interaction pattern persists for a long enough time, this will become apparent in a new pattern of aggregation. The transformation from one pattern of aggregation to another can be followed. If the interactions are too labile, there will be insufficient time for reaggregation to be observed, although our examples show rapid migration. In some such cases a useful strategy might be to segment the data and to restart the analysis process at those times when disaggregation is first detected.

Consider an experiment in which some number of stimuli (or behavioral conditions) recur cyclically. The data can be segmented on the stimulus boundaries, and a run of the analysis process can be carried out separately for each type of stimulus condition. The "pasting together" of the segments representing the same stimulus condition does not cause any boundary problem to our analysis process. In this guise we can address the question of whether different patterns of aggregation (representing particular values of neuronal interaction) are associated with different stimulus conditions. Other approaches to the problem of detecting time-varying coupling for two neurons subjected to repetitive stimulation are described by Gerstein and Perkel (1972) and by Schneider et al. (1983).

Generalization of charge and of particle dynamics. For concep. tual and computational simplicity we have assumed a single descending exponential function to describe the dynamics of "charge." We may generalize the definition and dynamics of charge to produce more appropriately shaped functions to produce performance more sensitive to particular types of neuronal interaction. For example, a low-order gamma function may replace the single exponential. It exhibits a single smooth maximum with a delay after spike time that may be chosen appropriately. Such functions would mimic known temporal properties of postsynaptic potentials. The modified charge function, $z_{i}$, would replace $q_{i}$ in equation 8 . However, in equation 7 , which defines the propulsive vector field, we would continue to use 
the $q_{i}$ as originally defined. The effect of this generalization is to enhance sensitivity to synaptic interactions having time courses similar to the new charge function. The reason for retaining the original $q_{j}$ in equation 7 is to associate synaptic effects upon other neurons with the firings of neuron $j$. Note that this modification means that both old and new charge functions must be calculated and maintained for all particles.

None of the above methodology explicitly addresses inhibitory influences among the observed neurons. Our basic technique may readily be generalized for this purpose. The idea is to redefine the second type of charge so as to reflect silence rather than firing. Such a charge function may be designed with the following properties: (1) it is small for short intervals; (2) it is small for long intervals; (3) it smoothly rises to a maximum for a selected intermediate interval length; (4) duration and shape within the interval are selected appropriately. This new charge again replaces $q_{i}$ in equation 8 rather than $q_{j}$ in equation 7 because we are selecting for the silent period of neuron $i$, which is putatively postsynaptically inhibited.

In the variations discussed so far, we cannot infer causal relations among the neurons represented by an aggregation of points. For certain classes of neuronal circuitry, this goal can be achieved through a further extension of these modifications. Specifically, we associate two points rather than one with each neuron. Each point bears one class of charge only; we refer to them as $q$-points and zpoints. In order to emphasize inhibitory interactions, we define the $z$-charge in terms of silences, as described above. We now specify two variations on the particle kinematics. In the first version, the $q$ points remain fixed at their original positions (at which they are mutually equidistant), while the $z$-points move as described by equations 7 and 8 , as modified by inclusion of the $z$-charge in equation 8 . With these rules, we can interpret an aggregation of $z$ points around a particular (distinct) $q$-point as direct evidence for divergent inhibition: the neuron corresponding to the q-point directly inhibits the neurons represented by the $z$-points clustering about it.

The second variation is the dual of the first. Here, $q$ is replaced by $z$ in equation 7 and not in equation 8 . Also, the $z$-points are constrained to their original position, and the q-points are allowed to migrate. We may interpret a cluster of $q$-points about a single zpoint as a convergent inhibitory circuit: the $z$-point corresponds to a neuron that receives inhibitory synaptic input from each of the neurons represented by the $q$-points clustered about it.

These variations are useful only after an initial survey of the neuronal population has been performed using the basic representation, i.e., using the original definition of charge and a single set of points. This initial screening can serve to identify appropriate subsets of the original neuronal population to which it will be useful to apply the types of variations described.

We emphasize that even these variations, which may use subsets of the original population, are performed in a higher-dimensional space, in which particle dynamics are governed by all interactions simultaneously. None of these approaches is equivalent to a set of pairwise comparisons, such as cross-correlation histograms (Perkel et al., 1967).
State of neuronal population. We have discussed our representation of neurons as migrating points in $\mathrm{N}$-space in the context of questions about functional circuitry in the neuronal population and about dynamic changes in that circuitry. However, this representation can also lead to a quantitative notion of the state of the neuronal population. By analogy with the phase-space representation used, for example, in statistical mechanics, we can abstract a still higherlevel representation than the one we have described. Specifically, we can incorporate the instantaneous positions and migration velocities of all $N$ points into a single vector, of dimension $2 N^{2}$. In an abstract space of this dimension, this vector is represented by a moving point. The trajectory of the point represents the temporal evolution of the state of the system, which in turn embodies the changing set of functional interactions among the neurons as manifested by their impulse activity.

For example, approximately closed or repeating trajectories of this state point would correspond to cyclic alterations in the organization of the neuronal population. Such changes in organization could arise from actual changes in effective connectivity as well as from alterations in thresholds, membrane excitability, and the like, or from changes in input external to the observed population of neurons.

We have mentioned these variations and extensions of our basic representation to emphasize that it is not merely a data-processing technique. On the contrary, it provides a unifying conceptual framework for understanding and depicting the activity of an entire neuronal population as an entity rather than as a collection of individual or pair properties. In particular, use of this type of representation can make concrete and measurable the notion of cell assembly that goes back to Hebb (1949). Further conceptual and computational development of this representation should prove fruitful.

\section{References}

Gerstein, G. L., and D. H. Perkel (1972) Mutual temporal relationships among neuronal spike trains. Biophys. J. 12: 453-473.

Gerstein, G. L., D. H. Perkel, and K. N. Subramanian (1978) Identification of functionally related neural assemblies. Brain Res. 140: 43-62.

Gerstein, G. L., M. J. Bloom, I. E. Espinosa, S. Evanczuk, and M. R. Turner (1983) Design of a laboratory for multineuron studies. IEEE Trans. Systerns, Man, and Cybernetics SMC-13: 668-676.

Grinvald, A., L. B. Cohen, S. Lesher, and M. B. Boyle (1981) Simultaneous optical monitoring of activity of many neurons in invertebrate ganglia using a 124 element photodiode array. J. Neurophysiol. 45: 829-840.

Hebb, D. O. (1949) The Organization of Behavior, John Wiley \& Sons, New York

Hubel, D. H., and T. N. Wiesel (1962) Receptive fields, binocular interaction and functional architecture in the cat's visual cortex. J. Physiol. (Lond.) 160: $106-154$

Mountcastle, V. B. (1957) Modality and topographic properties of single neurons of cat's somatic sensory cortex. J. Neurophysiol. 20: 408-434.

Perkel, D. H., G. L. Gerstein, and G. M. Moore (1967) Neuronal spike trains and stochastic point processes. II. Simultaneous spike trains. Biophys. J. 7: $419-440$.

Schneider, J., R. Eckhorn, and H. Reitboeck (1983) Evaluation of neuronal coupling dynamics. Biol. Cybernet. 46: 129-134.

Wright, W. E. (1977) Gravitational clustering. Pat. Recogn. 9: 151-166. 